\title{
PRODUCTS OF LIPSCHITZ-FREE SPACES AND APPLICATIONS
}

\author{
PEDRO L. KAUFMANN
}

\begin{abstract}
We show that, given a Banach space $X$, the Lipschitz-free space over $X$, denoted by $\mathcal{F}(X)$, is isomorphic to $\left(\sum_{n=1}^{\infty} \mathcal{F}(X)\right)_{\ell_{1}}$. Some applications are presented, including a non-linear version of Pełczyśki's decomposition method for Lipschitz-free spaces and the identification up to isomorphism between $\mathcal{F}\left(\mathbb{R}^{n}\right)$ and the Lipschitz-free space over any compact metric space which is locally bi-Lipschitz embeddable into $\mathbb{R}^{n}$ and which contains a subset that is Lipschitz equivalent to the unit ball of $\mathbb{R}^{n}$. We also show that $\mathcal{F}(M)$ is isomorphic to $\mathcal{F}\left(c_{0}\right)$ for all separable metric spaces $M$ which are absolute Lipschitz retracts and contain a subset which is Lipschitz equivalent to the unit ball of $c_{0}$. This class contains all $C(K)$ spaces with $K$ infinite compact metric (Dutrieux and Ferenczi had already proved that $\mathcal{F}(C(K))$ is isomorphic to $\mathcal{F}\left(c_{0}\right)$ for those $K$ using a different method). Finally we study Lipschitz-free spaces over certain unions and quotients of metric spaces, extending a result by Godard.
\end{abstract}

\section{INTRODUCTION}

Let $(M, d, 0)$ be a pointed metric space (that is, a distinguished point 0 in $M$, called base point, is chosen), and consider the Banach space $\operatorname{Lip}_{0}(M)$ of all real-valued Lipschitz functions on $M$ which vanish at 0, equipped with the norm

$$
\|f\|_{L i p}:=\inf _{x, y \in M, x \neq y} \frac{|f(x)-f(y)|}{d(x, y)} .
$$

On the closed unit ball of $\operatorname{Lip}_{0}(M)$, the topology of pointwise convergence is compact, so $\operatorname{Lip}_{0}(M)$ admits a canonical predual, which is called the Lipschitz-free space over $M$ and denoted by $\mathcal{F}(M)$. This space is the closure in $\operatorname{Lip}_{0}(M)^{*}$ of $\operatorname{span}\left\{\delta_{x}: x \in M\right\}$, where $\delta_{x}$ are the evaluation functionals defined by $\delta_{x}(f)=f(x)$. It is readily verified that $\delta: x \mapsto \delta_{x}$ is an isometry from $M$ into $\mathcal{F}(M)$. Given $0^{\prime}$, it is clear that $T: \operatorname{Lip}_{0}(M) \rightarrow \operatorname{Lip}_{0^{\prime}}(M)$ defined by $T(f):=f-f\left(0^{\prime}\right)$ is a weak*-to-weak* continuous isometric isomorphism, thus the choice of different base points yields isometrically isomorphic Lipschitz-free spaces. We refer to [14] for a study on Lipschitz functions spaces, and to [14] and [6] for an introduction to Lipschitz-free spaces and its basic properties.

One of the main properties of the Lipschitz-free spaces is that it permits to interpret Lipschitz maps between metric spaces from a linear point of view:

2010 Mathematics Subject Classification. Primary 46B20; Secondary 46 T99.

Key words and phrases. Lipschitz-free spaces, Geometry of Banach spaces, Spaces of Lipschitz functions. 
Proposition 1.1. Let $M$ and $N$ be pointed metric spaces, consider $\delta^{M}$ and $\delta^{N}$ the isometries that assign each $x \in M$ (respectively, each $x \in N$ ) to the corresponding evaluation functional $\delta_{x}^{M}$ in $\mathcal{F}(M)$ (respectively, $\delta_{x}^{N}$ in $\mathcal{F}(N)$ ), and suppose that $L: M \rightarrow N$ is a Lipschitz function such that $L\left(0_{M}\right)=0_{N}$. Then there is an unique linear map $\hat{L}: \mathcal{F}(M) \rightarrow \mathcal{F}(N)$ such that $\hat{L} \circ \delta^{M}=\delta^{N} \circ L$, that is, such that the following diagram commutes:

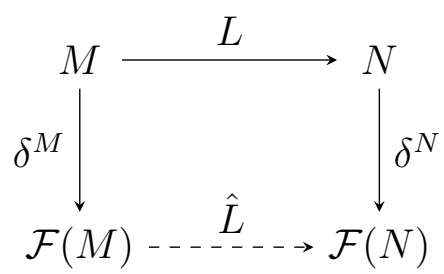

Moreover, $\|\hat{L}\|=\|L\|_{\text {Lip }}$.

In particular, if $M$ and $N$ are Lipschitz equivalent (that is, there is a bi-Lipschitz map between $M$ and $N$ ) then $\mathcal{F}(M)$ and $\mathcal{F}(N)$ are isomorphic. The converse is not true, even if $M$ and $N$ are assumed to be Banach spaces: if $K$ is an infinite compact metric space, then $\mathcal{F}(C(K))$ is isomorphic to $\mathcal{F}\left(c_{0}\right)$, even though $C(K)$ is not Lipschitz equivalent to $c_{0}$ in general (recall that, if $C(K)$ is uniformly homeomorphic to $c_{0}$, then it is isomorphic to $c_{0}$ c.f. [8]). This first counterexample for the Banach space case was presented by Dutrieux and Ferenczi in [3].

Despite of the simplicity of the definition of the Lipschitz-free spaces, many fundamental questions about their structure remain unanswered. Godard [5] characterized the metric spaces $M$ such that $\mathcal{F}(M)$ is isometrically isomorphic to a subspace of $L^{1}$ as exactly those who are isometrically embeddable into $\mathbb{R}$-trees (that is, connected graphs with no cycles with the graph distance); on the other hand, Naor and Schechtman [13] have shown that $\mathcal{F}\left(\mathbb{Z}^{2}\right)$ (thus also $\mathcal{F}\left(\mathbb{R}^{2}\right)$ ) is not isomorphic to any subspace of $L^{1}$. From this arises the natural question of characterizing the metric spaces $M$ such that $\mathcal{F}(M)$ is (nonisometrically) isomorphic to $L^{1}$. Godefroy and Kalton [6] have shown that, given a Banach space $X, X$ has the bounded approximation property if and only if $\mathcal{F}(X)$ has that property, and recently Hájek and Pernecká [4] have shown that $\mathcal{F}\left(\mathbb{R}^{n}\right)$ admits a Schauder basis, refining a result from [10]. However, it is not known whether $\mathcal{F}(F)$ admits a Schauder basis for any given closed subset $F \subset \mathbb{R}^{n}$. It is also not of this author's knowledge whether $\mathcal{F}\left(\mathbb{R}^{n}\right)$ is isomorphic to $\mathcal{F}\left(\mathbb{R}^{m}\right)$ or not, for distinct $m, n \geq 2$. Even the study of Lipschitz-free spaces over very simple subsets of $\mathbb{R}^{2}$ can present difficulties (see the question posed after Proposition 5.1.

In this context, we continue the exploration of what could be considered basic properties of Lipschitz-free spaces and their relation with the underlying metric spaces. We will show, for instance, that for any given Banach space $X$, we have that $\mathcal{F}(X)$ is isomorphic to $\left(\sum_{n=1}^{\infty} \mathcal{F}(X)\right)_{\ell_{1}}$ (see Theorem 3.1). This provides in particular a kind of non-linear version for Pełczyśki's decomposition method (see Corollary 3.2), which in turn can be used to obtain the mentioned example by Dutrieux and Ferenczi of non-Lipschitz equivalent Banach spaces sharing the same Lipschitz-free space. In fact, we show that $\mathcal{F}(M)$ is isomorphic 
to $\mathcal{F}\left(c_{0}\right)$ for a wider class of metric spaces (see Corollary 3.4). We will also show that, for compact metric spaces $M$ which are locally bi-Lipschitz embeddable in $\mathbb{R}^{n}$, we have that $\mathcal{F}(M)$ admits a complemented copy in $\mathcal{F}\left(\mathbb{R}^{n}\right)$; when moreover the euclidean ball $B_{\mathbb{R}^{n}}$ is bi-Lipschitz embeddable in $M$, we have that $\mathcal{F}(M)$ and $\mathcal{F}\left(\mathbb{R}^{n}\right)$ are actually isomorphic (see Theorem 3.7). The class of metric spaces satisfying both properties includes all $n$ dimensional compact Riemannian manifolds. Independently, we study the behavior of Lipschitz-free spaces with respect to certain gluings of metric spaces, expanding an initial idea presented by Godard [5].

1.1. Notation. We say that two metric spaces $M$ and $N$ are C-Lipschitz equivalent, for some constant $C>0$, if there a bi-Lipschitz onto map $\varphi: M \rightarrow N$ satisfying $\|\varphi\|_{L i p .}\left\|\varphi^{-1}\right\|_{L i p} \leq C . \quad M$ and $N$ are then Lipschitz equivalent if they are $C$-Lipschitz equivalent for some $C>0$; in that case we also write $M \stackrel{L}{\sim} N$. Given two Banach spaces $X$ and $Y$, we write $X \cong Y$ when $X$ and $Y$ are isometrically isomorphic, $X \stackrel{c}{\hookrightarrow} Y$ when there is a complemented copy of $X$ in $Y$, and $X \simeq Y$ when $X$ and $Y$ are isomorphic. If $X$ and $Y$ are isomorphic, the Banach-Mazur distance between $X$ and $Y$ is defined by

$$
d_{B M}(X, Y):=\inf \left\{\|T\| \cdot\left\|T^{-1}\right\|: T \text { is an isomorphism from } X \text { onto } Y\right\} .
$$

$\|T\| .\left\|T^{-1}\right\|$ is called the (linear) distortion of $T$. When $d_{B M}(X, Y) \leq C$ for some $C>0$, we say that $X$ is isomorphic to $Y$ with distortion bounded by $C$.

$\operatorname{Ext}_{0}(F, M)$ denotes the set of linear extension operators for Lipschitz functions and $\operatorname{Ext}_{0}^{p t}(F, M)$ is the set of pointwise-to-pointwise continuous elements of $\operatorname{Ext}_{0}(F, M)$ (see Subection 2.1).

1.2. Structure of this work. In Section 2, we present some background results on linear extension operators for Lipschitz functions and consequent ways to decompose the Lipschitz-free space over a metric space using metric quotients. In Section 3 we show that, for every Banach space $X, \mathcal{F}(X) \simeq\left(\sum_{n=1}^{\infty} \mathcal{F}(X)\right)_{\ell_{1}}$, and derive some consequences.

Sections 4 and 5 are independent of the results presented in Section 3. In Section 4 we show that, for every Banach space $X, d_{B M}\left(\mathcal{F}(X), \mathcal{F}(X) \oplus_{1} \mathcal{F}(X)\right) \leq 4$. In Section 5 we provide formulas for Lipschitz-free spaces over certain unions of metric spaces.

\section{LineAR EXTEnsions of Lipschitz FUnCTIONS AND THE LipsChitZ-FREE SPACE OVER METRIC QUOTIENTS}

2.1. Linear extensions of Lipschitz functions. Given a pointed metric space $(M, d, 0)$ and a subset $F$ containing 0 , let us denote by $\operatorname{Ext}_{0}(F, M)$ the set of all extensions $E$ : $\operatorname{Lip}_{0}(F) \rightarrow \operatorname{Lip}_{0}(M)$ which are linear and continuous $(E$ being an extension means that $\left.E(f)\right|_{F}=f$ for all $\left.f \in \operatorname{Lip}_{0}(F)\right)$. It is immediate to see that, if we choose another base point $0^{\prime}$ contained in $F$, to each element $E \in \operatorname{Ext}_{0}(F, M)$ there is a corresponding $E^{\prime} \in \operatorname{Ext}_{0^{\prime}}(F, M)$, defined by $E^{\prime}(f):=E\left(f-f\left(0^{\prime}\right)\right)+f\left(0^{\prime}\right)$, which satisfies $\left\|E^{\prime}\right\|=\|E\|$, so generally it is not important which base point is chosen. Recall that there are always 
continuous but not necessarily linear extensions from $\operatorname{Lip}_{0}(F)$ to $\operatorname{Lip}_{0}(M)$; for example the infimum convolution

$$
E(f)(x):=\inf _{y \in F}\left\{f(y)+\|f\|_{L i p} d(x, y)\right\}
$$

is such an extension and it is an isometry, although in most cases it fails to be linear. It is possible, though, to have $\operatorname{Ext}_{0}(F, M)=\emptyset$; Brudnyi and Brudnyi provide us with an example of a two-dimensional Riemannian manifold $M$, equipped with its geodesic metric, which admits a subset $F$ satisfying that condition (see Theorem 2.18 in [2]).

We will be particularly interested in the subset $\operatorname{Ext}_{0}^{p t}(F, M)$ of $\operatorname{Ext}_{0}(F, M)$ consisting of the pointwise-to-pointwise continuous elements. The fact that on bounded sets of $\operatorname{Lip}_{0}(F)$ the weak* and the pointwise topologies coincide implies that any element of $\operatorname{Ext}_{0}(F, M)$ is weak*-to-weak* continuous if and only if it belongs to $\operatorname{Ext}_{0}^{p t}(F, M)$. Therefore, any $E \in \operatorname{Ext}_{0}^{p t}(F, M)$ admits a preadjoint $P: \mathcal{F}(M) \rightarrow \mathcal{F}(F)$, which is a (continuous) canonical projection, in the sense that $P(\mu)=\left.\mu\right|_{F}$ for all finitely supported $\mu \in \mathcal{F}(M)$. In particular, $\mathcal{F}(F)$ is complemented in $\mathcal{F}(M)$. Reciprocally, given a continuous projection $P: \mathcal{F}(M) \rightarrow$ $\mathcal{F}(F)$ such that $P(\mu)=\left.\mu\right|_{F}$ for all finitely supported $\mu \in \mathcal{F}(M)$, we have that $P^{*} \in$ $\operatorname{Ext}_{0}^{p t}(F, M)$.

Even in the context where $M$ is a Banach space and $F$ is a closed linear subspace, we might not get this complementability condition. Consider, for example, $c_{0}$ and let $X$ be a subspace of $c_{0}$ which fails to have the bounded approximation property. As we mentioned in the introduction, given a Banach space $Y, Y$ has the bounded approximation property if and only if $\mathcal{F}(Y)$ has that property. Since this property is inherited by complemented subspaces, it follows that $\mathcal{F}(X)$ cannot be isomorphic to a complemented subspace of $\mathcal{F}\left(c_{0}\right)$. One can still pose the question of wether or not $\operatorname{Ext}_{0}\left(X, c_{0}\right)$ is empty.

On the other hand, we have the following positive example:

Proposition 2.1 (Lancien, Pernecká [10]). There exists $C>0$ such that, for each $n \in \mathbb{N}$ and each subset $F$ of $\mathbb{R}^{n}$ containing 0 , there exists $E$ in $\operatorname{Ext}_{0}^{p t}\left(F, \mathbb{R}^{n}\right)$ satisfying $\|E\| \leq$ $C \sqrt{n}$.

This result appears as part of the proof of Proposition 2.3 of [10], which states that $\mathcal{F}(F)$ has the $C \sqrt{n}$-bounded approximation property. It involves a construction by Lee and Naor from [12, and the fact that $\mathbb{R}^{n}$ admits a so-called $K$-gentle partition of the unity with respect to $F$, which in turn induces the mentioned extension $E$.

2.2. Metric quotients and Lipschitz-free spaces. We turn our attention to a special kind of metric quotient. Given a pointed metric space $(M, d, 0)$ and a subset $F$ of $M$ containing 0 , let $\sim$ be the equivalence relation which collapses $\bar{F}$ to a point (that is, the equivalence classes are either singletons or $\bar{F}$ ). We define the metric quotient of $M$ by $F$, denoted by $M / F$, as the pointed metric space $(M / \sim, \tilde{d},[0])$, where $\tilde{d}$ is defined by

$$
\tilde{d}([x],[y])=\min \{d(x, y), d(x, F)+d(y, F)\} .
$$

The space $\operatorname{Lip}_{[0]}(M / F)$ can be interpreted as the closed linear subspace of $\operatorname{Lip}_{0}(M)$ consisting of all of its functions which are null in $F$. Depending on how $F$ is placed in $M$, we can have the following decomposition for $\mathcal{F}(M)$ : 
Lemma 2.2. Let $(M, d, 0)$ be a pointed metric space and $F$ be a subset containing 0 , and suppose that there exists $E \in \operatorname{Ext}_{0}^{p t}(F, M)$. Then

$$
\mathcal{F}(M) \simeq \mathcal{F}(F) \oplus_{1} \mathcal{F}(M / F),
$$

with distortion bounded by $(\|E\|+1)^{2}$.

Proof. Define $\Phi: \operatorname{Lip}_{0}(F) \oplus_{\infty} \operatorname{Lip}_{0}(M / F) \rightarrow \operatorname{Lip}_{0}(M)$ by $\Phi(f, g) \doteq E(f)+g$. It is straightforward that $\Phi$ is an onto isomorphism with $\|\Phi\| \leq\|E\|+1$, that $\Phi$ is pointwiseto-pointwise continuous and that its inverse $\Phi^{-1}: h \mapsto\left(\left.h\right|_{F}, h-E\left(\left.h\right|_{F}\right)\right)$ has norm also bounded by $\|E\|+1$. It follows that $\Phi$ is the adjoint of an isomorphism $\Psi$ between $\mathcal{F}(M)$ and $\mathcal{F}(F) \oplus_{1} \mathcal{F}(M / F)$ satisfying the desired distortion bound.

\section{Products of Lipschitz-Free sPaCes}

In this section we will show that $\mathcal{F}(X) \simeq\left(\sum_{n=1}^{\infty} \mathcal{F}(X)\right)_{\ell_{1}}$ for any Banach space $X$, and derive some consequences. With that purpose we will use the following construction by Kalton [9]. Let $(M, d, 0)$ be a pointed metric space, denote by $B_{r}$ the closed balls centered at 0 and with radius $r>0$ and consider, for each $k \in \mathbb{Z}$, the linear operator $T_{k}: \mathcal{F}(M) \rightarrow \mathcal{F}\left(B_{2^{k+1}} \backslash B_{2^{k-1}}\right)$ defined by

$$
T_{k} \delta_{x}:= \begin{cases}0, & \text { if } x \in B_{2^{k-1}} \\ \left(\log _{2} d(x, 0)-k+1\right) \delta_{x}, & \text { if } x \in B_{2^{k} \backslash B_{2^{k-1}}} \\ \left(k+1-\log _{2} d(x, 0)\right) \delta_{x}, & \text { if } x \in B_{2^{k+1}} \backslash B_{2^{k}} \\ 0, & \text { if } x \notin B_{2^{k+1}} .\end{cases}
$$

Lemma 4.2 from [9] says that for each $\gamma \in \mathcal{F}(M)$ we have that $\gamma=\sum_{k \in \mathbb{Z}} T_{z} \gamma$ unconditionally and

$$
\sum_{k \in \mathbb{Z}}\left\|T_{k} \gamma\right\|_{\mathcal{F}} \leq 72\|\gamma\|_{\mathcal{F}}
$$

Another result from that same paper that we will use is Lemma 4.2, which states that, given $r_{1}, \ldots, r_{n}, s_{1}, \ldots, s_{n} \in \mathbb{Z}, r_{1}<s_{1}<r_{2}<\cdots<s_{n}$ and $\gamma_{k} \in \mathcal{F}\left(B_{2^{s_{k}}} \backslash B_{2^{r_{k}}}\right)$ and writing $\theta:=\min _{k=1, \ldots, n-1}\left\{r_{k+1}-s_{k}\right\}$, then

$$
\left\|\gamma_{1}+\cdots+\gamma_{n}\right\|_{\mathcal{F}} \geq \frac{2^{\theta}-1}{2^{\theta}+1} \sum_{k=1}^{n}\left\|\gamma_{k}\right\|_{\mathcal{F}} .
$$

Theorem 3.1. Let $X$ be a Banach space. Then

$$
\mathcal{F}(X) \simeq\left(\sum_{n=1}^{\infty} \mathcal{F}(X)\right)_{\ell_{1}} .
$$

Proof. Note that $S:\left(\gamma_{k}\right) \in\left(\sum_{k \in \mathbb{Z}} \mathcal{F}\left(B_{2^{k+1}} \backslash B_{2^{k-1}}\right)\right)_{\ell_{1}} \mapsto \sum_{k \in \mathbb{Z}} \gamma_{k} \in \mathcal{F}(X)$ is linear, continuous and onto, and from (3.1) we get that $T: \gamma \in \mathcal{F}(X) \mapsto\left(T_{k} \gamma\right) \in\left(\sum_{k \in \mathbb{Z}} \mathcal{F}\left(B_{2^{k+1}} \backslash\right.\right.$ $\left.\left.B_{2^{k-1}}\right)\right)_{\ell_{1}}$ is a well defined one-to-one linear continuous operator. Thus $T \circ S$ is a continuous projection from $\left(\sum_{k \in \mathbb{Z}} \mathcal{F}\left(B_{2^{k+1}} \backslash B_{2^{k-1}}\right)\right)_{\ell_{1}}$ onto the isomorphic copy $T(\mathcal{F}(X))$ of $\mathcal{F}(X)$. 
Denote $M:=\cup_{k \in \mathbb{Z}}\left(B_{2^{2 k+1}} \backslash B_{2^{2 k}}\right)$, and consider $E \in \operatorname{Ext}_{0}(M \cup\{0\}, X)$ which extends each element of $\operatorname{Lip}_{0}(M \cup\{0\})$ linearly on each radial segment $\left[2^{2 k-1}, 2^{2 k}\right] x, k \in \mathbb{Z}, x \in S_{X}$. Clearly $E$ is pointwise-to-pointwise continuous, thus it is the adjoint of some $P: \mathcal{F}(X) \rightarrow$ $\mathcal{F}(M \cup\{0\})$, which is a projection which satisfies $P(\mu)=\left.\mu\right|_{M}$ for all finitely supported $\mu \in \mathcal{F}(X)$. Note that $\mathcal{F}(M \cup\{0\}) \cong \mathcal{F}(M)$, since $0 \in \bar{M}$. Now by 3.2 , the natural identification $I d: \mathcal{F}(M) \rightarrow\left(\sum_{k \in \mathbb{Z}} \mathcal{F}\left(B_{2^{2 k+1}} \backslash B_{2^{2 k}}\right)\right)_{\ell_{1}}$ is an isomorphism. So there is a complemented copy of $\left(\sum_{k \in \mathbb{Z}} \mathcal{F}\left(B_{2^{2 k+1}} \backslash B_{2^{2 k}}\right)\right)_{\ell_{1}}$ in $\mathcal{F}(X)$.

Note that, by Proposition 1.1, re-scalings of any metric space give rise to isometrically isomorphic Lipschitz-free spaces. Thus all spaces $\mathcal{F}\left(B_{2^{2 k+1}} \backslash B_{2^{2 k}}\right), k \in \mathbb{Z}$ are isometrically isomorphic to $\mathcal{F}\left(B_{2} \backslash B_{1}\right)$ and all spaces $\mathcal{F}\left(B_{2^{k+1}} \backslash B_{2^{k-1}}\right), k \in \mathbb{Z}$ are isometrically isomorphic to $\mathcal{F}\left(B_{4} \backslash B_{1}\right)$, which in turn is isomorphic to $\mathcal{F}\left(B_{2} \backslash B_{1}\right)$. It follows that

$$
\mathcal{F}(X) \stackrel{c}{\hookrightarrow}\left(\sum_{j=1}^{\infty} \mathcal{F}\left(B_{2} \backslash B_{1}\right)\right)_{\ell_{1}} \text { and }\left(\sum_{j=1}^{\infty} \mathcal{F}\left(B_{2} \backslash B_{1}\right)\right)_{\ell_{1}} \stackrel{c}{\hookrightarrow} \mathcal{F}(X) \text {. }
$$

Since $\left(\sum_{j=1}^{\infty} \mathcal{F}\left(B_{2} \backslash B_{1}\right)\right)_{\ell_{1}}$ is isomorphic to its $\ell_{1}$-sum, by a standard Pełczyński's decomposition method we have

$$
\mathcal{F}(X) \simeq\left(\sum_{j=1}^{\infty} \mathcal{F}\left(B_{2} \backslash B_{1}\right)\right)_{\ell_{1}}
$$

and the conclusion follows immediately.

As a direct consequence of Theorem 3.1 and Proposition 1.1, we get the following nonlinear version of Pełczyński's decomposition method for Lipschitz-free spaces.

Corollary 3.2. Let $X$ be a Banach space and $M$ be a metric space, and suppose that $X$ and $M$ admit Lipschitz retracts $N_{1}$ and $N_{2}$, respectively, such that $X$ is Lipschitz equivalent to $N_{2}$ and $M$ is Lipschitz equivalent to $N_{1}$. Then $\mathcal{F}(X) \simeq \mathcal{F}(M)$.

Proof. $\mathcal{F}(X)$ is isomorphic to $\mathcal{F}\left(N_{2}\right)$, which in turn is a complemented subspace of $\mathcal{F}(M)$. Analogously, $\mathcal{F}(M)$ is isomorphic to a complemented subspace of $\mathcal{F}\left(N_{1}\right)$. The conclusion follows by applying the standard Pełczyński's decomposition method.

Corollary 3.3. Let $X$ be a Banach space. Then

$$
\mathcal{F}(X) \simeq \mathcal{F}\left(B_{1}\right)
$$

Proof. Since $B_{1}$ is a Lipschitz retract of $X$, it follows that $\mathcal{F}(X)$ contains a complemented copy of $\mathcal{F}\left(B_{1}\right)$. In the proof of Theorem 3.1 we have shown that $\mathcal{F}(X)$ is isomorphic to $\left(\sum_{k \in \mathbb{Z}} \mathcal{F}\left(B_{2^{2 k+1}} \backslash B_{2^{2 k}}\right)\right)_{\ell_{1}}$, which is clearly isomorphic to $\left(\sum_{k<0} \mathcal{F}\left(B_{2^{2 k+1}} \backslash B_{2^{2 k}}\right)\right)_{\ell_{1}}$ since all summands are isometrically isomorphic. Let $N:=\cup_{k<0}\left(B_{2^{2 k+1}} \backslash B_{2^{2 k}}\right)$ Again by (3.2), $\left(\sum_{k<0} \mathcal{F}\left(B_{2^{2 k+1}} \backslash B_{2^{2 k}}\right)\right)_{\ell_{1}}$ is isomorphic to $\mathcal{F}(N)$, which is complemented in $\mathcal{F}\left(B_{1}\right)$ since there is a pointwise-to-pointwise continuous element in $\operatorname{Ext}_{0}\left(N \cup\{0\}, B_{1}\right)$. The conclusion 
follows by an application of Pełczyński's decomposition method.

Recall that a subset $F$ of a metric space $M$ is called a Lipschitz retract of $M$ if there is a Lipschitz map from $M$ onto $F$ which coincides with the identity on $F$; in case such a map exists, it is called a Lipschitz retraction. A metric space is said to be an absolute Lipschitz retract if it is a Lipschitz retract of every metric space containing it. Given any metric space $M$, the space $C_{u}(M)$ of real-valued bounded and uniformly continuous functions on $M$, equipped with the uniform norm, is an example of Banach space which is an absolute Lipschitz retract (see e.g. [1], Theorem 1.6). This class includes all $C(K)$ spaces for $K$ compact metric space, in particular it includes $c_{0}$. Since all separable metric spaces are bi-Lipschitz embeddable in $c_{0}$ ([1], Theorem 7.11), we obtain the following class of metric spaces $M$ with $\mathcal{F}(M) \simeq \mathcal{F}\left(c_{0}\right)$ :

Corollary 3.4. Let $M$ be a separable metric space containing a Lipschitz retract which is Lipschitz equivalent to the unit ball of $c_{0}$, and suppose that $M$ is an absolute Lipschitz retract. Then $\mathcal{F}(M) \simeq \mathcal{F}\left(c_{0}\right)$.

In particular, if $K$ is an infinite compact metric space, then $\mathcal{F}(C(K)) \simeq \mathcal{F}\left(c_{0}\right)$.

Proof. It is straightforward by Proposition 1.1 and Corollary 3.3 that there is a complemented copy of $\mathcal{F}\left(c_{0}\right)$ in $\mathcal{F}(M)$. $M$ is Lipschitz equivalent to some subset $F$ of $c_{0}$, and $F$ is an absolute Lipschitz retract since this property is preserved by Lipschitz equivalences. Thus $F$ is a Lipschitz retract of $c_{0}$, and again by Proposition 1.1 this implies that $\mathcal{F}(F)$ (and thus $\mathcal{F}(M)$ ) admits a complemented copy in $\mathcal{F}\left(c_{0}\right)$. The conclusion follows from Theorem 3.1 and an application of Pełczyński's decomposition method.

Corollary 3.5. Let $F$ be a subset of $\mathbb{R}^{n}$ with nonempty interior. Then $\mathcal{F}(F) \simeq \mathcal{F}\left(\mathbb{R}^{n}\right)$.

Proof. By Proposition 2.1, there is a complemented copy of $\mathcal{F}(F)$ in $\mathcal{F}\left(\mathbb{R}^{n}\right)$. Taking any closed ball $B$, it is easy to see that there is a Lipschitz retraction from $F$ onto $B$; thus by Proposition 1.1 and Corollary 3.3 there is also a complemented copy of $\mathcal{F}\left(\mathbb{R}^{n}\right)$ in $\mathcal{F}(F)$. The result follows from Theorem 3.1 and an application of Pełczyński's decomposition method. .

Remark. In [4], Hájek and Pernecká have shown that $\mathcal{F}\left(\mathbb{R}^{n}\right)$ admits a Schauder basis, and rose the natural question of whether or not the same holds true for $\mathcal{F}(F)$, being $F$ any closed subset of $\mathbb{R}^{n}$. Note that, by Corollary 3.5 , the problem is reduced to the case where $F$ has empty interior.

In order to study Lipschitz-free spaces of locally euclidean metric spaces, alongside the corollaries of Theorem 3.1, the following result becomes handy:

Theorem 3.6 (Lang, Plaut [1] ). Let $M$ be a compact metric space such that each point of $M$ admits a neighborhood which is bi-Lipschitz embeddable in $\mathbb{R}^{n}$. Then $M$ is bi-Lipschitz embeddable in $\mathbb{R}^{n}$. 
Theorem 3.7. Let $M$ be a compact metric space such that each $x \in M$ admits a neighborhood which is bi-Lipschitz embeddable in $\mathbb{R}^{n}$. Then there is a complemented copy of $\mathcal{F}(M)$ in $\mathcal{F}\left(\mathbb{R}^{n}\right)$.

If moreover the unit ball of $\mathbb{R}^{n}$ is bi-Lipschitz embeddable into $M$, then $\mathcal{F}(M) \simeq \mathcal{F}\left(\mathbb{R}^{n}\right)$. In particular, the Lipschitz-free space over any n-dimensional compact Riemannian manifold equipped with its geodesic metric is isomorphic to $\mathcal{F}\left(\mathbb{R}^{n}\right)$.

Proof. The first part follows directly from Lang and Plaut's result and the fact that the Lipschitz-free space over any subset of $\mathbb{R}^{n}$ admits a complemented copy in $\mathcal{F}\left(\mathbb{R}^{n}\right)$.

For the second part, note that the closed unit ball of $\mathbb{R}^{n}$ is an absolute Lipschitz retract, and recall that that property is preserved by Lipschitz equivalences. The result then follows from Corollary 3.5. Theorem 3.1 and an application of Pełczyński's decomposition method.

Remark. Note that the compactness condition in Theorem 3.7 is necessary, even if have uniformity on the embeddings into $\mathbb{R}^{n}$. For example, $\mathbb{Z} \times \mathbb{R}$ is locally isometric to line segments, but $\mathcal{F}(\mathbb{Z} \times \mathbb{R})$ is not isomorphic to a subspace of $\mathcal{F}(\mathbb{R}) \cong L^{1}$, by Naor and Schechtman's result mentioned in the introduction.

\section{4. $\mathcal{F}(X) \simeq \mathcal{F}(X)^{2}$ WITH LOW DISTORTION}

Let $X$ be a Banach space. By Theorem 3.1, $\mathcal{F}(X) \simeq \mathcal{F}(X)^{2}$. In this Section we will show that we have the uniform bound $d_{B M}\left(\mathcal{F}(X), \mathcal{F}(X) \oplus_{1} \mathcal{F}(X)\right) \leq 4$; we will do this via an elementary construction based on metric properties of $X$.

We start by recalling some definitions and results on quotient metric spaces which are of a more general kind than the ones presented in Section 2. For details and more on that subject, we refer to Weaver's book [14]. Let $(M, d)$ be a complete metric space, and let $\sim$ be an equivalence relation on $M$. The element of $M / \sim$ containing $x \in M$ will be denoted by either $\tilde{x}$ or $[x]_{\sim}$. Define a pseudometric $\tilde{d}$ on $M / \sim$ by

$$
\tilde{d}(\tilde{x}, \tilde{y}):=\inf \left\{\sum_{j=1}^{n} d\left(x_{j}, y_{j}\right): n \in \mathbb{N}, x \sim x_{1}, y_{j} \sim x_{j+1}(j=1, \ldots, n-1), y_{n} \sim y\right\} .
$$

This pseudometric can be roughly interpreted in the following way: it is the length of the shortest discrete path from $x$ to $y$ when we are allowed to teleport between equivalent elements. An equivalent way to define $\tilde{d}$, that will be useful for further constructions, is the following:

$$
\tilde{d}(\tilde{x}, \tilde{y})=\sup \left\{|f(x)-f(y)|: f: M \rightarrow \mathbb{R} \text { is constant in each } \tilde{z} \in \tilde{M},\|f\|_{\text {Lip }} \leq 1\right\},
$$

where $\|f\|_{\text {Lip }}$ is the Lipschitz constant of $f$.

On $M$ we define yet the equivalence relation $\approx$ which identifies all $x, y \in M$ satisfying $\tilde{d}(\tilde{x}, \tilde{y})=0$, and on $M / \approx$ we define the metric $\tilde{\tilde{d}}(\tilde{\tilde{x}}, \tilde{\tilde{y}})=\tilde{d}(\tilde{x}, \tilde{y})$. We define $M_{\sim}$, the metric quotient (or just quotient) of $M$ with respect to $\sim$, as the completion of $M / \approx$. Note that, for a given complete metric space $(M, d, 0)$ and an equivalence relation $\sim$ on $M$, by 4.2 
there is a canonical isometric isomorphism between $\operatorname{Lip}_{\tilde{\tilde{0}}}\left(M_{\sim}\right)$ and the closed subspace of $\operatorname{Lip}_{0}(M)$ consisting of all functions that are constant in each class $\tilde{x} \in M / \sim$.

We recall some definitions concerning path metric spaces. Let $(M, d)$ be a pseudometric space, and let $\varphi: I \rightarrow M$ be a curve (that is, $I$ is an interval and $\varphi$ is continuous). The length of $\varphi$ is $\ell(\varphi):=\sup \left\{\sum_{j=1}^{n} d\left(\varphi\left(x_{j-1}\right), \varphi\left(x_{j}\right)\right)\right\}$, where the supremum is taken over $n \in \mathbb{N}$ and $x_{j} \in I, x_{0}<\cdots<x_{n} .(M, d)$ is said to be a path metric space if $d$ is a metric and $d(x, y)=\inf \{\ell(\varphi): \varphi$ is a curve in $M$ having endpoints $x$ and $y\}$. A minimizing geodesic in a path metric space is any curve $\varphi: I \rightarrow M$ such that $d(\varphi(t), \varphi(s))=|t-s|$ for all $t, s \in I ;(M, d)$ is said to be geodesic if any two elements of $M$ are joined by a minimizing geodesic.

Proposition 4.1. Let $(M, d)$ be a path metric space. Then each metric quotient of $M$ is a path metric space.

Proof. Fix an equivalence relation $\sim$ on $M$. Let $x, y \in M$, and for each $k \in \mathbb{N}$ consider pairs $\left(x_{1}^{k}, y_{1}^{k}\right), \ldots,\left(x_{n_{k}}^{k}, y_{n_{k}}^{k}\right)$ of elements of $M$ such that

$$
x \sim x_{1}^{k}, y_{j}^{k} \sim x_{j+1}^{k}\left(j=1, \ldots, n_{k}-1\right), y_{n_{k}}^{k} \sim y
$$

and $\sum_{j=1}^{n_{k}} d\left(x_{j}^{k}, y_{j}^{k}\right) \stackrel{k}{\rightarrow} \tilde{d}(\tilde{x}, \tilde{y})$. Since $(M, d)$ is a path metric space, there exist, for each $k \in \mathbb{N}$ and $j=1, \ldots, n_{k}$, curves $\varphi_{j}^{k}$ with endpoints $x_{j}^{k}$ and $y_{j}^{k}$, respectively, and such that

$$
\sum_{j=1}^{n_{k}} \ell\left(\varphi_{j}^{k}\right)<\tilde{d}(\tilde{x}, \tilde{y})+\frac{1}{k} .
$$

Concatenating we get a curve $\tilde{\varphi}^{k}$ in $M / \sim$ with endpoints $\tilde{x}$ and $\tilde{y}$ satisfying $\ell\left(\tilde{\varphi}^{k}\right)<$ $\tilde{d}(\tilde{x}, \tilde{y})+\frac{1}{k}$. Since for any curve $\tilde{\varphi}$ in $M / \sim$ with endpoints $\tilde{x}$ and $\tilde{y}$ we have $\tilde{d}(\tilde{x}, \tilde{y}) \leq \ell(\tilde{\varphi})$, it follows that

$$
\tilde{d}(\tilde{x}, \tilde{y})=\inf \{\ell(\tilde{\varphi}): \tilde{\varphi} \text { is a curve in } M / \sim \text { having endpoints } \tilde{x} \text { and } \tilde{y}\},
$$

and then clearly the same holds for $M / \approx$ and thus for $M_{\sim}$.

Remark: In Proposition 4.1 we cannot substitute path metric space by geodesic metric space:

Proposition 4.2. There is a geodesic metric space $M$ which admits a metric quotient that is a non-geodesic path metric space.

Proof. Let $e_{j}$ be the standard unit vectors of $\ell_{1}$ and consider the metric subspace of $\ell_{1}$ defined by $M:=\cup_{j=1}^{\infty}[0,1] e_{j}$. Let $F:=\cup_{j=1}^{\infty}\left\{e_{j}\right\}$ and suppose that $\sim$ is the equivalence relation which collapses $F$ to a point. Note that, in this case, $M / \sim=M_{\sim}$, that the $M_{\sim^{-}}$ distance between $\tilde{0}$ and $\tilde{e_{1}}=F$ is 1 and that there are minimizing geodesics with endpoints $\tilde{0}$ and $\tilde{e_{1}}$ going through each segment $[0,1] e_{j}$.

Let $F_{j}:=\left[\frac{1}{4}+\frac{1}{2^{2+j}}, \frac{3}{4}-\frac{1}{2^{2+j}}\right] e_{j}$ be interpreted as a subset of $M$, and consider on $M$ the equivalence relation $\equiv$ that collapses each $F_{j}$ to a point, and the respective quotient metric space $(M / \equiv, d)$ (again, in this case we have $\left.M_{\equiv}=M / \equiv\right)$. Then $d\left([\tilde{0}]_{\equiv},\left[\tilde{e_{1}}\right]_{\equiv}\right)=\frac{1}{2}$ and 
there are curves $\varphi_{j}$ in $M / \equiv$ with endpoints $[\tilde{0}]_{\equiv}$ and $\left[\tilde{e}_{1}\right]_{\equiv}$ with $\ell\left(\varphi_{j}\right) \stackrel{j}{\rightarrow} \frac{1}{2}$, even though there is no minimizing geodesic in $M / \equiv$ with endpoints $[\tilde{0}]_{\equiv}$ and $\left[\tilde{e_{1}}\right]_{\equiv}$. One verifies the path metric property for all other pairs of elements of $M / \equiv$.

Lemma 4.3. Consider $M$ a path metric space, $N$ a metric space, $f: M \rightarrow N$ and $C>0$. Then $f$ is C-Lipschitz if and only if it is locally C-Lipschitz.

Proof. To prove the nontrivial implication, fix $\delta>0$, let $x, y \in M$ and let $\varphi: I \rightarrow M$ be a curve with endpoints $x$ and $y$ satisfying

$$
\ell(\varphi)<d_{M}(x, y)+\delta .
$$

For each $t \in I$, there exists by hypothesis $\epsilon_{t}>0$ such that $\left.f\right|_{\varphi(] t-\epsilon_{t}, t+\epsilon_{t}[)}$ is $C$-Lipschitz. Since $I$ is compact, there are $t_{1}<\cdots<t_{n}$ such that $\left.\cup_{j=1}^{\infty}\right] t_{j}-\epsilon_{t_{j}}, t_{j}+\epsilon_{t_{j}}[\supset I$. We can then easily find $\varphi$-consecutive points $z_{1}, \ldots, z_{m}$ in $\varphi(I)$ satisfying

$$
\begin{aligned}
d_{N}(f(x), f(y)) & \leq d_{N}\left(f(x), f\left(z_{1}\right)\right)+d_{N}\left(f\left(z_{1}\right), f\left(z_{2}\right)\right)+\cdots+d_{N}\left(f\left(z_{m}\right), f(y)\right) \\
& \leq C\left(d_{M}\left(x, z_{1}\right)+d_{M}\left(z_{1}, z_{2}\right)+\cdots+d_{M}\left(z_{m}, y\right)\right) \\
& \leq C\left(d_{M}(x, y)+\delta\right) .
\end{aligned}
$$

Since $\delta$ was arbitrary, the conclusion follows.

Let $(X,\|\cdot\|)$ be a Banach space. We now proceed with the construction of a pair of metric quotients of $X$, namely $X_{L}$ and $X_{R}$, which have properties useful for studying products of $\operatorname{Lip}_{0}(X)$ (see Proposition 4.6). Let $\alpha, \beta:[0,+\infty) \rightarrow[0,+\infty)$ be the continuous functions defined in each $\left[2^{m}, 2^{m+1}\right], m \in \mathbb{Z}$ by

$$
\alpha(t):= \begin{cases}t-2^{m-1}, & \text { if } 2^{m} \leq t \leq 2^{m-1}+2^{m} \\ 2^{m}, & \text { if } 2^{m-1}+2^{m} \leq t \leq 2^{m+1}\end{cases}
$$

and

$$
\beta(t):= \begin{cases}2^{m-1}, & \text { if } 2^{m} \leq t \leq 2^{m-1}+2^{m} \\ t-2^{m}, & \text { if } 2^{m-1}+2^{m} \leq t \leq 2^{m+1} .\end{cases}
$$

Consider the equivalence relations $\sim_{L}$ and $\sim_{R}$ on $X$ defined by

$$
x \sim_{L} y \Leftrightarrow x=y \text { or }(x=\lambda y \text { with } \lambda>0, \text { and } \alpha \text { is constant in }[\|x\|,\|y\|])
$$

and

$$
x \sim_{R} y \Leftrightarrow x=y \text { or }(x=\lambda y \text { with } \lambda>0, \text { and } \beta \text { is constant in }[\|x\|,\|y\|])
$$

and denote by $X_{L}=\left(X_{L}, d_{L}\right)$ and $X_{R}=\left(X_{R}, d_{R}\right)$ the corresponding quotient metric spaces.

To prove the next lemma we use Hopf-Rinow's Theorem which states that in a complete and locally compact path metric space, each pair of points are joined by a minimizing geodesic (see e.g. [7]).

Lemma 4.4. $X_{L}$ and $X_{R}$ are geodesic. 


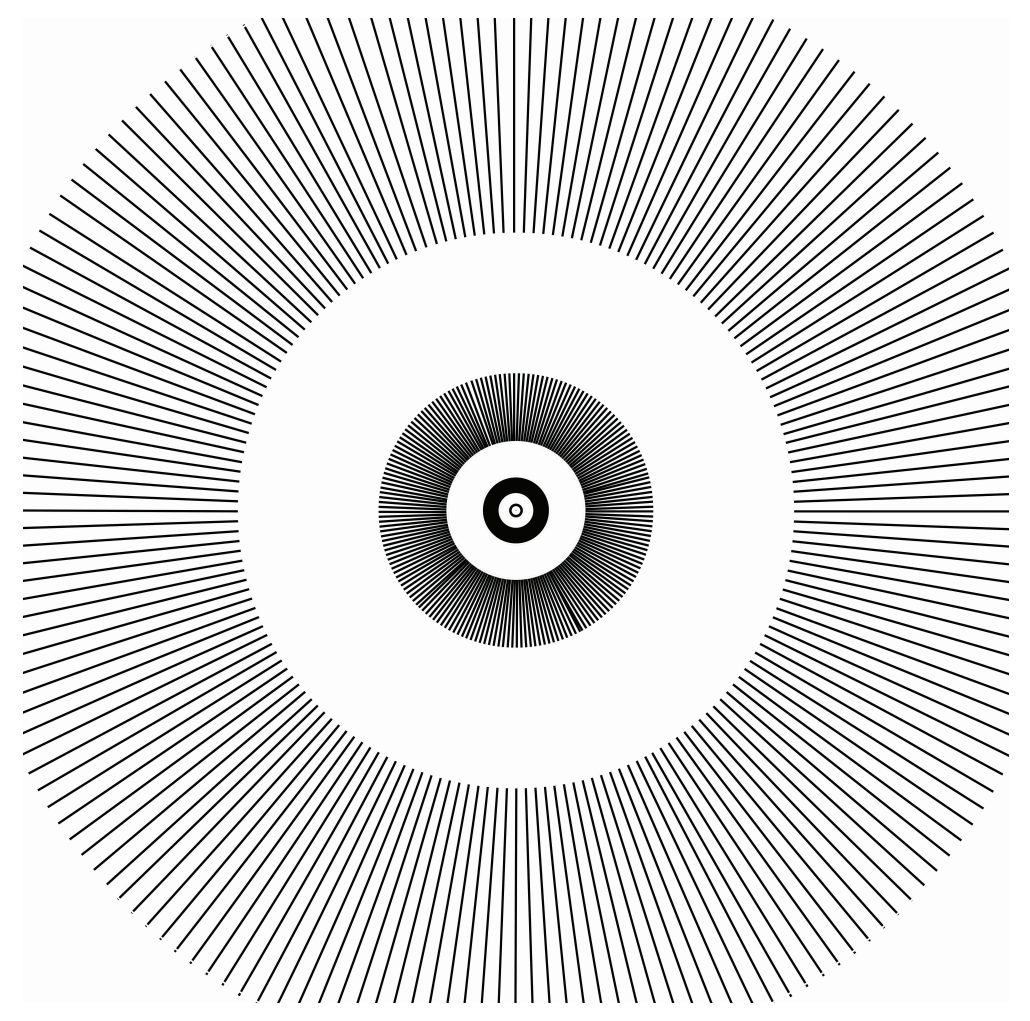

Figure 1. This is how $X_{L}$ looks like. The represented radial segments are collapsed to points. $X_{R}$ looks the same, up to a factor two re-scaling.

Proof. For any $x, y \in X$, note that the metric space $\left(\operatorname{span}_{X}\{x, y\} / \sim_{R}, d_{R}\right)$ satisfies the conditions in Hopf-Rinow's Theorem, thus there is a minimizing geodesic $\gamma$ in $\left(\operatorname{span}_{X}\{x, y\} / \sim_{R}, d_{R}\right.$ ) (thus also in $X_{R}$ ) with endpoints $\tilde{x}$ and $\tilde{y}$. The same argument holds for $X_{L}$.

Lemma 4.5. There exist onto bi-Lipschitz mappings $L: X \rightarrow X_{L}$ and $R: X \rightarrow X_{R}$ with $\|L\|_{\text {Lip }} \leq 1,\left\|L^{-1}\right\|_{\text {Lip }} \leq \frac{4}{3},\|R\|_{\text {Lip }} \leq \frac{3}{2}$ and $\left\|R^{-1}\right\|_{\text {Lip }} \leq 1$.

Proof. Denote $C_{m}:=B_{2^{m+1}} \backslash B_{2^{m}}, N \in \mathbb{N}$, and for each $x \in X \backslash\{0\}$ let $m_{x} \in \mathbb{Z}$ be such that $\|x\| \in C_{m_{x}}$. Define the bicontinuous mapping $R: X \backslash\{0\} \rightarrow X_{R} \backslash\{\tilde{0}\}$ by

$$
R(x):=\left(\left(\frac{1}{2}+\frac{2^{m_{x}}}{\|x\|}\right) x\right)^{\sim_{R}} .
$$

What $R$ does is to squeeze each crown $C_{m}$ onto the thinner crown $R\left(C_{m}\right)=\left(B_{2^{m+1}} \backslash\right.$ $\left.B_{2^{m}+2^{m-1}}\right)_{R}$. For $x \in X \backslash\{0\}$, let $V_{x}$ be a neighborhood of $x$ such that, for each $y \in V_{x}$, $\|x-y\| \leq 2^{m_{x}-1}$ and $d_{R}(R(x), R(y)) \leq 2^{m_{x}-2}$. This will imply that, for any $y \in V_{x}$, we have $\left|m_{x}-m_{y}\right| \leq 1$, the line segment with endpoints $x$ and $y$ intercepts at most two crowns $C_{m}$ and a minimizing geodesic with endpoints $R(x)$ and $R(y)$ intercepts at most 
two crowns $R\left(C_{m}\right)$. We shall show that

$$
\|x-y\| \leq d_{R}(R(x), R(y)) \leq \frac{3}{2}\|x-y\|, x \in X \backslash\{0\}, y \in V_{x} .
$$

The fact that $X$ and $X_{R}$ have minimizing geodesics connecting any pair of points will allow us then to assert, by Lemma 4.3, that the above inequality holds without the restriction $y \in V_{x}$, and thus that $R$ is bi-Lipschitz, $\|R\|_{\text {Lip }} \leq \frac{3}{2}$ and $\left\|R^{-1}\right\|_{\text {Lip }} \leq 1$.

In effect, let $x, y \in X \backslash B_{m}$ with $\|x-y\| \leq 2^{m-1}$ and $d_{R}(R(x), R(y)) \leq 2^{m-2}$. Assume without loss of generality that $\|x\| \leq\|y\|$. Then one of the following conditions is true:

(1) $m_{x}=m_{y}$, and $d_{R}(R(x), R(y))=\|R(x)-R(y)\|$;

(2) $m_{x}<m_{y}$

(3) $m_{x}=m_{y}$, and there is a minimizing geodesic with endpoints $R(x)$ and $R(y)$ passing through $R\left(C_{m_{x}-1}\right)$.

If (1) is true, then $\frac{1}{2}+\frac{2^{m_{x}}}{\|y\|} \leq \frac{1}{2}+\frac{2^{m_{x}}}{\|x\|}$, and

$$
\left(\frac{1}{2}+\frac{2^{m_{x}}}{\|y\|}\right)\|x-y\| \leq\|R(x)-R(y)\| \leq\left(\frac{1}{2}+\frac{2^{m_{x}}}{\|x\|}\right)\|x-y\|,
$$

thus

$$
\|x-y\| \leq d_{R}(R(x), R(y)) \leq \frac{3}{2}\|x-y\| .
$$

If (2) is true, suppose that $\|x\|<2^{m_{x}+1}$ (if $\|x\|=2^{m_{x}+1}$, then $x$ and $y$ satisfy (1)) and let $z$ be the intersection point between the line segment $[x, y]$ and $S_{2^{m_{x}+1}}$. Then the pairs $x, z$ and $z, y$ satisfy (1), and by (4.4) we have

$$
d_{R}(R(x), R(y)) \leq d_{R}(R(x), R(z))+d_{R}(R(z), R(y)) \leq \frac{3}{2}(\|x-z\|+\|z-y\|)=\frac{3}{2}\|x-y\| .
$$

Similarly, let $\tilde{z}$ be the intersection of $R\left(S_{2^{m_{x}+1}}\right)$ with a minimizing geodesic with endpoints $R(x)$ and $R(y)$. Then $d_{R}(R(x), \tilde{z})=\|R(x)-\tilde{z}\|$ and $d_{R}(\tilde{z}, R(y))=\|\tilde{z}-R(y)\|$, and thus by (4.4) we have that

$$
\|x-y\| \leq\left\|x-R^{-1}(\tilde{z})\right\|+\left\|R^{-1}(\tilde{z})-y\right\| \leq d_{R}(R(x), \tilde{z})+d_{R}(\tilde{z}, R(y))=d_{R}(R(x), R(y)) .
$$

For the remainder case (3), we can obtain the desired inequalities by taking a convenient point in a minimizing geodesic with endpoints $R(x)$ and $R(y)$ and reducing the problem to the case $(2)$.

The Lipschitz equivalence between $X$ and $X_{L}$ is given by the mapping $L: X \backslash\{0\} \rightarrow$ $X_{L} \backslash\{\tilde{0}\}$ defined by

$$
L(x):=\left(\left(\frac{1}{2}+\frac{2^{m_{x}-1}}{\|x\|}\right) x\right)^{\sim_{L}},
$$

which squeezes each crown $C_{m}$ onto the thinner crown $L\left(C_{m}\right)=\left(B_{2^{m}+2^{m-1}} \backslash B_{2^{m}}\right)^{\sim_{L}}$. To show this and obtain the Lipschitz constants, one simply must follow the same steps taken 
to do that for $R$. The only difference will appear when getting to 4.3), which will read

$$
\left(\frac{1}{2}+\frac{2^{m_{x}-1}}{\|y\|}\right)\|x-y\| \leq\|L(x)-L(y)\| \leq\left(\frac{1}{2}+\frac{2^{m_{x}-1}}{\|x\|}\right)\|x-y\|,
$$

and thus 4.4 will read

$$
\frac{3}{4}\|x-y\| \leq d_{L}(L(x), L(y)) \leq\|x-y\| .
$$

Following analogous steps, we get to the conclusion.

Proposition 4.6. Let $X$ be a Banach space. Then $d_{B M}\left(\mathcal{F}(X), \mathcal{F}(X) \oplus_{1} \mathcal{F}(X)\right) \leq 4$.

Proof. Recall that, by (4.2), $\operatorname{Lip}_{0}\left(X_{L}\right) \cong Y_{L}$ and $\operatorname{Lip}_{0}\left(X_{R}\right) \cong Y_{R}$, where $Y_{L}$ and $Y_{R}$ are the closed subspaces of $\operatorname{Lip}_{0}(X)$ defined by

$$
Y_{L}:=\left\{f \in \operatorname{Lip}_{0}(X): f \text { is constant in each equivalence class of } X_{L}\right\}
$$

and

$$
Y_{R}:=\left\{f \in \operatorname{Lip}_{0}(X): f \text { is constant in each equivalence class of } X_{R}\right\} .
$$

Let $\Phi: Y_{L} \oplus_{\infty} Y_{R} \rightarrow \operatorname{Lip}_{0}(X)$ be defined by $\Phi(f, g):=f+g$. Then $\Phi$ is linear with norm $\|\Phi\| \leq 2$. Moreover, $\Phi$ admits an inverse defined by

$$
\left(\Phi^{-1} h\right)(x)=\left(\frac{\alpha(\|h(x)\|)}{\|h(x)\|} h(x), \frac{\beta(\|h(x)\|)}{\|h(x)\|} h(x)\right) .
$$

Since the functions $x \mapsto \frac{\alpha(\|x\|)}{\|x\|} x$ and $x \mapsto \frac{\beta(\|x\|)}{\|x\|} x$ are 1-Lipschitz, it follows that $\left\|\Phi^{-1}\right\| \leq 1$. Now from Lemma 4.5 it follows that there is an isomorphism $\Psi$ from $\operatorname{Lip}_{0}(X) \oplus_{\infty} \operatorname{Lip}_{0}(X)$ onto $Y_{L} \oplus_{\infty} Y_{R}$ satisfying $\|\Psi\| \cdot\left\|\Psi^{-1}\right\| \leq \frac{4}{3} \cdot \frac{3}{2}=2$. Then $\Phi \circ \Psi$ is an isomorphism from $\operatorname{Lip}_{0}(X) \oplus_{\infty} \operatorname{Lip}_{0}(X)$ onto $\operatorname{Lip}_{0}(X)$ satisfying $\|\Phi \circ \Psi\| \cdot\left\|(\Phi \circ \Psi)^{-1}\right\| \leq 4$. Since $\Phi$ and $\Psi$ are pointwise-to-pointwise continuous, $\Phi \circ \Psi$ induces an isomorphism $T: \mathcal{F}(X) \rightarrow$ $\mathcal{F}(X) \oplus_{1} \mathcal{F}(X)$ satisfying $T^{*}=\Phi \circ \Psi$ and $\|T\| \cdot\left\|T^{-1}\right\| \leq 4$

\section{ON LIPSCHITZ-FREE SPACES OVER UNIONS OF METRIC SPACES}

In this section we will provide a couple of formulas for computing the Lipschitz-free space over certain unions of metric spaces from the Lipschitz-free spaces of the original metric spaces, provided that we have an orthogonal placement of the metric spaces involved (in a sense to be made precise). The first one generalizes in particular a first step taken in this direction by Godard [5] (see Proposition 5.2 below). The idea of studying the behavior of the Lipschitz-free spaces with respect to unions is motivated, for example, by the problem of characterizing the metric spaces such that the corresponding Lipschitz-free spaces admit isomorphic embeddings into $L^{1}$. We start by studying how taking certain orthogonal unions of metric spaces have effect on the corresponding Lipschitz-free spaces:

Proposition 5.1. Suppose that $M=\cup_{\gamma \in \Gamma} M_{\gamma}$ is a metric space with metric d, and suppose that there exists $0 \in M$ satisfying 
(1) $M_{\gamma} \cap M_{\eta}=\{0\}$ for $\gamma \neq \eta$, and

(2) (orthogonality) there exists $C \geq 1$ such that, for all $\gamma \neq \eta, x \in M_{\gamma}$ and $y \in M_{\eta}$, $d(x, 0)+d(y, 0) \leq C d(x, y)$.

Then

$$
\mathcal{F}\left(\bigcup_{\gamma \in \Gamma} M_{\gamma}\right) \simeq\left(\sum_{\gamma \in \Gamma} \mathcal{F}\left(M_{\gamma}\right)\right)_{\ell_{1}}
$$

with distortion bounded by $C$.

Proof. We can assume $0=0_{M}=0_{M_{\gamma}}$, for all $\gamma$. Consider

$$
\Phi:\left(f_{\gamma}\right) \in\left(\sum_{\gamma \in \Gamma} \operatorname{Lip}_{0}\left(M_{\gamma}\right)\right)_{\ell_{\infty}} \mapsto h \in \operatorname{Lip}_{0}\left(\cup_{\gamma \in \Gamma} M_{\gamma}\right),
$$

where $\left.h\right|_{M_{\gamma}}=f_{\gamma} . h=\Phi\left(f_{\gamma}\right)$ is well defined since, for each $x \in M_{\zeta}$ and $y \in M_{\eta}$ with $\zeta \neq \eta$,

$$
\begin{aligned}
\left|\Phi\left(\left(f_{\gamma}\right)\right)(x)-\Phi\left(\left(f_{\gamma}\right)\right)(y)\right| & =\left|f_{\zeta}(x)-f_{\eta}(y)\right| \leq\left\|f_{\zeta}\right\| d(x, 0)+\left\|f_{\eta}\right\| d(y, 0) \\
& \leq C \max \left\{\left\|f_{\zeta}\right\|,\left\|f_{\eta}\right\|\right\} d(x, y) \leq C\left\|\left(f_{\gamma}\right)\right\| d(x, y) .
\end{aligned}
$$

Since $\Phi$ is linear, we have from the inequality above that

$$
\|\Phi\| \leq \max \{1, C\} .
$$

It is clear that $\Phi$ is surjective, and that $\left\|\Phi^{-1}\right\| \leq 1$. $\Phi$ is weak*-to-weak* continuous, thus it is the adjoint of an isomorphism from $\mathcal{F}\left(\cup_{\gamma \in \Gamma} M_{\gamma}\right)$ onto $\left(\sum_{\gamma \in \Gamma} \mathcal{F}\left(M_{\gamma}\right)\right)_{\ell_{1}}$. It is clear from (5.2) that the distortion of $\Phi$ is bounded by $C$.

Note that, if we exclude the orthogonality condition, the concatenating operator $\Phi$ defined in (5.1) does not need to take values in $\operatorname{Lip}_{0}(M)$. We do not know if it is possible to remove this condition, even for finite unions. In fact, in particular we do not now the answer to the following apparently simple question:

Problem. Consider the set Cusp $:=\{(x, 0): x \geq 0\} \cup\left\{\left(x, x^{2}\right): x \geq 0\right\}$ endowed with the euclidean metric. Is $\mathcal{F}(C u s p)$ isomorphic to a subspace of $L^{1}$ ?

Note that Cusp is not Lipschitz equivalent to the real line, so we cannot appeal to Proposition 1.1 to have a positive answer. A negative answer would imply that there is no isomorphic copy of $\mathcal{F}\left(\mathbb{R}^{2}\right)$ in $L^{1}$, which as we mentioned is one of the main results from [13].

The mentioned result by Godard can be seen as a particular case of Proposition 5.1:

Proposition 5.2 (Godard [5, Proposition 5.1). Let $\Gamma$ be a set with a distiguished point 0 , denote $\Gamma^{*}:=\Gamma \backslash\{0\}$, and let $M=\cup_{\gamma \in \Gamma} M_{\gamma}$ be a metric space with metric d. Suppose that 
there exist $A, B>0$ such that $A \leq d(x, y) \leq B$ whenever $x$ and $y$ belong to different $M_{\gamma}$ 's. Then

$$
\mathcal{F}(M) \simeq\left(\sum_{\gamma \in \Gamma} \mathcal{F}\left(M_{\gamma}\right)\right)_{\ell_{1}} \oplus \ell_{1}\left(\Gamma^{*}\right)
$$

(Alternative) proof. Assume that $A \leq 1 \leq B$. Fix a point $p \in M_{0}$, and for each $\gamma \neq 0$ fix $0_{\gamma} \in M_{\gamma}$ and define $\Phi_{\gamma}=\left(\Phi_{\gamma}^{1}, \Phi_{\gamma}^{2}\right): \operatorname{Lip}_{p}\left(M_{\gamma} \cup\{p\}\right) \rightarrow \operatorname{Lip}_{0_{\gamma}}\left(M_{\gamma}\right) \oplus_{\infty} \mathbb{R}$ by

$$
\left(\Phi_{\gamma}^{1}(f), \Phi_{\gamma}^{2}(f)\right):=\left(f-f\left(0_{\gamma}\right), f\left(0_{\gamma}\right)\right) .
$$

It is easily seen that $\left\|\Phi_{\gamma}\right\| \leq B$. $\Phi_{\gamma}$ admits an inverse $\Phi_{\gamma}^{-1}$ which is defined by $\Phi_{\gamma}^{-1}(f, r)(x)=$ $f(x)+r$, if $x \in M_{\gamma}$ and $\Phi_{\gamma}^{-1}(f, r)(p)=0$. We have the bound $\left\|\Phi_{\gamma}^{-1}\right\| \leq \frac{B+1}{A}$; to see this, let $f \in B_{\text {Lip }_{0_{\gamma}}\left(M_{\gamma}\right)}$ and $r \in \mathbb{R}$ with $|r| \leq 1$, and let $x \in M_{\gamma}$. Then

$$
\frac{\left|\Phi_{\gamma}^{-1}(f, r)(x)-\Phi_{\gamma}^{-1}(f, r)(p)\right|}{d(x, p)} \leq \frac{|f(x)+r|}{d(x, p)} \leq \frac{|f(x)|+|r|}{A} \leq \frac{B+1}{A} .
$$

It follows that $\Phi: \operatorname{Lip}_{p}\left(M_{0}\right) \oplus_{\infty}\left(\sum_{\gamma \in \Gamma^{*}} \operatorname{Lip}_{p}\left(M_{\gamma} \cup\{p\}\right)\right)_{\ell_{\infty}} \rightarrow\left(\sum_{\gamma \in \Gamma} \operatorname{Lip}_{0_{\gamma}}\left(M_{\gamma}\right)\right)_{\ell_{\infty}} \oplus_{\infty}$ $\ell_{\infty}\left(\Gamma^{*}\right)$ defined by

$$
\Phi\left(f,\left(f_{\gamma}\right)_{\gamma \in \Gamma^{*}}\right):=\left(\left(f,\left(\Phi_{\gamma}^{1}\left(f_{\gamma}\right)\right)_{\gamma \in \Gamma^{*}}\right),\left(\Phi_{\gamma}^{2}\left(f_{\gamma}\right)\right)_{\gamma \in \Gamma^{*}}\right)
$$

is a pointwise-to-pointwise continuous isomorphism with $\|\Phi\| \cdot\left\|\Phi^{-1}\right\| \leq \frac{B(B+1)}{A}$. It is therefore the adjoint of an isomorphism

$$
\mathcal{F}\left(M_{0}\right) \oplus_{1}\left(\sum_{\gamma \in \Gamma^{*}} \mathcal{F}\left(M_{\gamma} \cup\{p\}\right)\right)_{\ell_{1}} \simeq\left(\sum_{\gamma \in \Gamma} \mathcal{F}\left(M_{\gamma}\right)\right)_{\ell_{1}} \oplus_{1} \ell_{1}\left(\Gamma^{*}\right) .
$$

Now the spaces $M_{0},\left(M_{\gamma} \cup\{p\}\right), \gamma \in \Gamma^{*}$ satisfy the orthogonality condition (2) of Proposition 5.1. thus $\mathcal{F}(M)=\mathcal{F}\left(M_{0} \cup\left(\cup_{\gamma \in \Gamma^{*}}\left(M_{\gamma} \cup\{p\}\right)\right) \simeq \mathcal{F}\left(M_{0}\right) \oplus_{1}\left(\sum_{\gamma \in \Gamma^{*}} \mathcal{F}\left(M_{\gamma} \cup\{p\}\right)\right)_{\ell_{1}}\right.$, and the conclusion follows by $(5.3)$.

Note that, using Lemma 2.2, we can also have some control when the intersection of the metric spaces involved is nontrivial. We present a version for the union of two metric spaces.

Proposition 5.3. Let $M \cup N$ be a metric space with metric d, suppose that $F:=M \cap N$ is closed and nonempty, and choose a base point 0 in F. Assume that

(1) there exists $E$ in $\operatorname{Ext}_{0}^{p t}(F, M \cup N)$ satisfying $\|E\| \leq C$, and

(2) (orthogonality) there exists $C \geq 1$ such that, for all $x \in M$ and $y \in N, d(x, F)+$ $d(y, F) \leq C d(x, y)$.

Then

$$
\mathcal{F}(M \cup N) \simeq \mathcal{F}(M / F) \oplus_{1} \mathcal{F}(N / F) \oplus_{1} \mathcal{F}(F)
$$

with distortion bounded by $C(\|E\|+1)^{2}$. 
Proof. Denote by $\tilde{d}$ the metric of $(M \cup N) / F$, and note that $M / F$ and $N / F$ are subsets of $(M \cup N) / F$. For each $\tilde{x} \in M / F$ and each $\tilde{y} \in N / F$,

$$
\begin{aligned}
\tilde{d}(\tilde{x}, \tilde{0})+\tilde{d}(\tilde{y}, \tilde{0}) & =\min \{d(x, 0), d(x, F)\}+\min \{d(y, 0), d(y, F)\} \\
& =d(x, F)+d(y, F) \leq C \min \{d(x, y), d(x, F)+d(y, F)\}=C \tilde{d}(\tilde{x}, \tilde{y})
\end{aligned}
$$

and thus we can apply Proposition 5.1 and get that $d_{B M}\left(\mathcal{F}(M \cup N / F), \mathcal{F}(M / F) \oplus_{1}\right.$ $\mathcal{F}(N / F)) \leq C$. Since $\mathcal{F}(M \cup N)$ is $(\|E\|+1)^{2}$-isomorphic to $\mathcal{F}((M \cup N) / F) \oplus_{1} \mathcal{F}(F)$ by Lemma 2.2 , the result follows.

Acknowledgements This research was supported by CAPES, grant BEX 10057/12-9.

I am grateful for having been warmly welcomed by the Équipe d'Analyse Fonctionnelle during my postdoctoral period at the IMJ. I am especially grateful to Gilles Godefroy for many fruitful discussions which lead to several improvements of this work. I thank Florent Baudier for driving my attention to [11]. I thank Bruno Braga, Marek Cúth and Michal Doucha for reading my original manuscript and pointing out flaws to be corrected. Illustration by Gabriela Kaufmann Sacchetto.

\section{REFERENCES}

[1] Beniyamini, Lindenstrauss, Geometric Nonlinear Functional Analysis, vol. 1, Amer. Math. Soc. Coll. Publ., vol. 48, Amer. Math. Soc., Providence, 2000. (2012) , 1135-1148.

[2] A. Brudnyi and Y. Brudnyi, Metric spaces with linear extensions preserving Lipschitz condition, Amer. J. Math., 129(1) (2007), 217-314.

[3] Y. Dutrieux and V. Ferenczi, The Lipschitz free Banach spaces of $C(K)$-spaces, Proc. Amer. Math. Soc., 134(4) (2006), 1039-1044.

[4] P. Hájek and E. Pernecká, On Schauder bases in Lipschitz-free spaces, preprint.

[5] A. Godard, Tree metrics and their Lipschitz-free spaces, Proc. Amer. Math. Soc., 138 (2010), 43114320.

[6] G. Godefroy and N.J. Kalton, Lipschitz-free Banach spaces, Studia Math., 159(1) (2003), 121-141.

[7] M. Gromov, Metric Structures for Riemannian and Non-Riemannian Spaces, Springer, 2001.

[8] W. B. Johnson, J. Lindenstrauss and G. Schechtman, Banach spaces determined by their uniform structures, Geom. Funct. Anal., 6(3) (1996), 430-470.

[9] N. J. Kalton, Spaces of Lipschitz and Hölder functions and their applications, Collect. Math., 55(2) (2004), 171-217.

[10] G. Lancien and E. Pernecká, Approximation properties and Schauder decompositions in Lipschitz-free spaces, J. Func. Anal. 264(10) (2013), 2323-2334.

[11] U. Lang and C. Plaut, Bilipschitz embeddings of metric spaces into space forms, Geom. Dedicata, 87 (2001), 285-307.

[12] J. R. Lee and A. Naor, Extending Lipschitz functions via random metric partitions, Inv. Math., 160 (2005), 59-95.

[13] A. Naor and G. Schechtman, Planar earthmover is not in $L^{1}$, SIAM J. Comput., 37(3) (2007), $804-826$.

[14] N. Weaver, Lipschitz Algebras, World Scientific Publishing Co. Inc., River Edge, NJ, 1999. 
CAPES Foundation, Ministry of Education of Brazil, Brasília/DF 70040-020, Brazil and Institute de Mathématiques de Jussieu, Université Pierre et Marie Curie, 4 Place Jussieu, 75005 Paris, France 